\title{
ANALYTIC SOLUTIONS OF THE VECTOR BURGERS' EQUATION
}

\author{
BY \\ STEVEN NERNEY ( NASA-Marshall Space Flight Center, AL and Ohio University, Lancaster) \\ EDWARD J. SCHMAHL (Department of Astronomy, University of Maryland, College Park) \\ AND
}

Z. E. MUSIELAK (Dept. of Mechanical and Aerospace Engineering, Univ. of Alabama at Huntsville)

\begin{abstract}
The well-known analytical solution of Burgers' equation is extended to curvilinear coordinate systems in three dimensions by a method that is much simpler and more suitable to practical applications than that previously used [22]. The results obtained are applied to incompressible flow with cylindrical symmetry, and also to the decay of an initially linearly increasing wind.
\end{abstract}

1. Introduction. Burgers' equation is a well-known example of a nonlinear partial differential equation whose solution can be constructed from a linear partial differential equation. It is, to the best of our knowledge, the only such example. Much of the interest in this equation arises because it is a very simple form of the Navier-Stokes equation in the one-dimensional, Cartesian, time-dependent, compressible, viscous limit [17]. An early study derived two steady-state solutions [1], and then the equation was used in studying the decay of free turbulence $[4,5]$. The importance of the equation is due to the nonlinear term, $u \partial u / \partial x$, which allows the calculation of the modification of the velocity due to the exchange of momentum between a variety of different length scales. Burgers' equation is the simplest type that shows the complicated interplay between the nonlinear steepening and the diffusion of a wave.

The first full solution of the one-dimensional Burgers' equation was found independently by both Cole [7] and Hopf [12]. Recently, the solution has been extended to $n$ dimensions by using group action on coset bundles [22]. The basic idea of this approach is to determine coset spaces for a chosen group and for a chain of closed subgroups, and then to construct a bundle out of these spaces. Next, the action of the group on the bundle is prescribed and a class of tensor-valued functions called invariants on the coset bundle is introduced. Because these group invariants take the form of differential equations, the method has been used to obtain a tensor version of the $n$-dimensional Burgers' equation and to show that its solutions can be con-

Received May 17, 1993.

1991 Mathematics Subject Classification. Primary 35K99.

(C)1996 Brown University 
structed from a linear tensor diffusion equation. It has also been demonstrated that the procedure gives a nonlinear tensor equation for a generalized Burgers' equation with a higher than second order derivative. In this paper, we examine extensions of the basic class of solutions of the vector Burgers' equation, which we discovered before we became aware of the work of Wolf et al., to more than one dimension and to curvilinear coordinate systems. The main advantage of our approach is that it is extremely simple from a mathematical point of view and that the results we obtain have the potential to be used in a number of applications (see Section III for two simple applications). It must be added, however, that the solutions obtained in this paper represent a subclass of more general solutions to the tensor Burgers' equation discussed by Wolf et al. [22]. In what follows, we recapitulate part of the historical derivation presented first by Cole [7] and Hopf [12]. This derivation is central to our results in Sections II and III.

The equation to be solved is

$$
\frac{\partial u}{\partial t}+u \frac{\partial u}{\partial x}=\nu \frac{\partial^{2} u}{\partial x^{2}}
$$

where $\nu$ is a physical constant (viscosity). A successful transformation uses

$$
u=\frac{\partial \phi}{\partial x}, \quad \phi=\phi(x, t)
$$

Substituting into Eq. (1) and integrating term-by-term with respect to $x$,

$$
\frac{\partial \phi}{\partial t}+\frac{1}{2}\left(\frac{\partial \phi}{\partial x}\right)^{2}=\nu \frac{\partial^{2} \phi}{\partial x^{2}}
$$

where the function of integration is omitted. Because Eq. (3) is invariant under the transformation

$$
\begin{aligned}
x & \rightarrow a x, \\
t & \rightarrow a^{2} t,
\end{aligned}
$$

( $a=$ constant $)$, as is the diffusion equation, this suggests that there exists a solution of the form

$$
\phi(x, t)=\phi(\theta(x, t))
$$

where $\theta$ is a solution of the diffusion equation

$$
\frac{\partial \theta}{\partial t}=\nu \frac{\partial^{2} \theta}{\partial x^{2}}
$$

Substitution into Eq. (3) while subtracting Eq. (6) gives

$$
\left(\frac{\partial \phi}{\partial \theta}\right)^{2}=2 \nu \frac{\partial^{2} \phi}{\partial \theta^{2}}
$$

whose solution is

$$
\phi(\theta)=-2 \nu \ln \left(\theta-c_{1}\right)+c_{2},
$$

so that, by choosing $c_{1}=0$,

$$
u(x, t)=\frac{-2 \nu}{\theta} \frac{\partial \theta}{\partial x} .
$$


Quite an interesting literature has developed around applications of the solutions in pure mathematics [20], magnetohydrodynamics [9], astrophysics [14, 16], and cosmology $[10,15]$. Lighthill [18] extended the basic transformation to the case where the $x$-coordinate is measured in the frame of reference moving in the same direction as the wave at the undisturbed speed of sound. A valuable book on Burgers' equation and related topics was recently published but is, unfortunately, already out of print [11].

In the following, we formally present the derivation of the solutions to the vector Burgers' equation by using a generalized Cole-Hopf transformation.

2. The vector extension of Burgers' equation. We begin with the vector equivalent of Burgers' equation

$$
\frac{\partial \vec{u}}{\partial t}+\vec{u} \cdot \nabla \vec{u}=\nu \nabla^{2} \vec{u} .
$$

In order to evaluate the vector Laplacian we must use

$$
\nabla^{2} \vec{u}=\nabla(\nabla \cdot \vec{u})-\nabla \times \nabla \times \vec{u},
$$

and for the inertial term

$$
\vec{u} \cdot \nabla \vec{u}=\nabla \frac{u^{2}}{2}-\vec{u} \times \nabla \times \vec{u} .
$$

Then Eq. (9) greatly simplifies as long as

$$
\vec{u} \times \nabla \times \vec{u}=\nu \nabla \times \nabla \times \vec{u} .
$$

This appears to be an equation that represents quite general flows, but the only solutions are irrotational. This can be seen by taking the divergence of both sides of Eq. (10c) and using

$$
\nabla \cdot(\vec{A} \times \vec{B})=\vec{B} \cdot \nabla \times \vec{A}-\vec{A} \cdot \nabla \times \vec{B} .
$$

In what follows we will assume that the flow field is irrotational since this is necessary to simplify Eq. (9) and to derive the extension of the Cole-Hopf transformation. The assumption is satisfied trivially for the one-dimensional Cartesian problem

$$
\nabla \times \vec{u}=0,
$$

so that

$$
\vec{u}=\nabla \phi .
$$

Eq. (11b) is the extension of Eq. (2) to three dimensions and, together with Eqs. (10), allows the simplification of Eq. (9) to

$$
\nabla\left[\frac{\partial \phi}{\partial t}+\frac{(\nabla \phi)^{2}}{2}-\nu \nabla^{2} \phi\right]=0 .
$$

The meaning of Eq. (12) is that the quantity enclosed by brackets must be a function of time, only, because its gradient is zero in all space so that

$$
\frac{\partial \phi}{\partial t}+\frac{(\nabla \phi)^{2}}{2}-\nu \nabla^{2} \phi=E(t)
$$


The form of the unknown function $E(t)$ is irrelevant for our discussion since it cannot affect the velocity. We simply define a new potential

$$
\phi_{1}=\phi-\int E(t) d t,
$$

where the gradients of $\phi$ and $\phi_{1}$ are identical.

Before introducing the Cole-Hopf assumption, it is useful to state the following vector identity:

$$
\nabla \alpha=\frac{\partial \alpha}{\partial \theta} \nabla \theta
$$

which is true for any orthogonal, curvilinear coordinate system. Taking the divergence of both sides with $\alpha=\phi$ and using Eq. (14a) again with $\alpha=\frac{\partial \phi}{\partial \theta}$ yields

$$
\nabla^{2} \phi=\frac{\partial^{2} \phi}{\partial \theta^{2}}(\nabla \theta)^{2}+\frac{\partial \phi}{\partial \theta} \nabla^{2} \theta
$$

We will also need the diffusion equation

$$
\frac{\partial \theta}{\partial t}=\nu \nabla^{2} \theta
$$

Now we apply Eqs. (5), (14b), and (15) to Eq. (13) to generate Eq. (7a), again, so that

$$
\vec{u}=-\frac{2 \nu}{\theta} \nabla \theta .
$$

This clearly shows that the Cole-Hopf transformation goes through for quite general coordinate systems. Cole [7] originally discussed the vector solution for the case of Cartesian coordinates, and he indicated that it applied to some special solutions in higher dimensions.

The usefulness of Eq. (16) can be shown by exploiting the simple symmetry of the following example.

3. Cylindrical symmetry. Using only a radial velocity, Burgers' equation in cylindrical coordinates with axisymmetry may be written as

$$
\frac{\partial u}{\partial t}+u \frac{\partial u}{\partial r}=\nu\left[\frac{1}{r} \frac{\partial}{\partial r}\left(r \frac{\partial u}{\partial r}\right)-\frac{u}{r^{2}}\right]
$$

We initially noticed that (17) could be written as

$$
\frac{\partial u}{\partial t}+u \frac{\partial u}{\partial r}=\nu \frac{\partial}{\partial r}\left(\frac{1}{r} \frac{\partial}{\partial r}(r u)\right)
$$

Equation (18) and the related integrals of (18) are what led us to believe that the Cole-Hopf transformation was quite general in nature. The particular form of (16) is

$$
u=\frac{-2 \nu}{\theta} \frac{\partial \theta}{\partial r}
$$

where the diffusion equation is

$$
\frac{\partial \theta}{\partial t}=\frac{\nu}{r} \frac{\partial}{\partial r}\left(r \frac{\partial \theta}{\partial r}\right)
$$


A simple example of the use of this method is to use the initial-value solution of the diffusion equation (a Dirac delta at $t=r=0$ )

$$
\theta=\frac{1}{2 \nu t} e^{-\frac{r^{2}}{4 \nu t}}
$$

Then the solution of (17) is found from (19),

$$
u=\frac{r}{t} \text {. }
$$

This is the cylindrical version of Burgers' Cartesian solution [4], $u=x / t$, which is the inviscid solution of (1) (solution 2.1, figure 5 in Benton [3]). Because (22) satisfies (17) in the limit as $\nu \rightarrow 0$ (which is also the case of zero acceleration for fluid particles), this solution is the inviscid limit of the solution of Burgers' equation in cylindrical coordinates. Some solutions of (17), such as example A below, approach the sawtooth limit as $t \rightarrow \infty$; but this is not surprising since it has been noted [2, 13] that the sawtooth shape of dissipation layers is of fundamental importance in Burgers' representation of turbulence.

It is extremely useful to derive solutions of the diffusion equation that are based on physical boundary conditions on the velocity field. To this end, Eq. (19) may be formally integrated to

$$
\theta(r, t)=k(t) \exp \left(-\frac{1}{2 \nu} \int_{0}^{r} u(w, t) d w\right)
$$

where

$$
k(t)=\theta(0, t) .
$$

The velocity is to be initially specified over a given range so that the initial value of $\theta$ is given by

$$
\theta(r, 0)=\theta_{0}(r)=k_{0} \exp \left(-\frac{1}{2 \nu} \int u_{0}(w) d w\right)
$$

for $r_{0} \leq r \leq R$.

The general solution of the diffusion equation, (20), with azimuthal symmetry, is [19]

$$
\theta(r, t)=\int_{0}^{\infty} \int_{0}^{\infty} \theta_{0}\left(r^{\prime}\right) J_{0}\left(k r^{\prime}\right) J_{0}(k r) e^{-\nu k^{2} t} k r^{\prime} d k d r^{\prime}
$$

The integral over $k$ can be done once and for all since it is a special case of Weber's second exponential integral as discussed in Watson [21]:

$$
\int_{0}^{\infty} \exp \left(-\nu t k^{2}\right) J_{0}(k r) J_{0}\left(k r^{\prime}\right) k d k=\frac{1}{2 \nu t} \exp \left(-\frac{r^{2}+r^{\prime 2}}{4 \nu t}\right) I_{0}\left(\frac{r r^{\prime}}{2 \nu t}\right)
$$

where the modified Bessel function of order zero may be written as

$$
I_{0}\left(\alpha r^{\prime}\right)=J_{0}\left(i \alpha r^{\prime}\right),
$$

which is a real function. Equation (26) may now be written as

$$
\theta(r, t)=\frac{1}{2 \nu t} \exp \left(-\frac{r^{2}}{4 \nu t}\right) \int_{0}^{\infty} \theta_{0}\left(r^{\prime}\right) \exp \left(-\frac{r^{\prime 2}}{4 \nu t}\right) J_{0}\left(\frac{i r^{\prime} r}{2 \nu t}\right) r^{\prime} d r^{\prime}
$$


4. Two simple examples. We choose to work two relatively simple examples so as not to obscure the simple nature of the transform. It is surprisingly easy to pose apparently straight-forward problems that cannot be readily carried to completion because of extremely difficult quadratures. We will treat the more difficult case of the decay of an initially constant velocity wind in a future paper.

The reader may have noticed by now that Burgers' equation does not specify the density distribution except in a few limits besides the trivial one of constant density (as in the next example).

A. Incompressible flow. The first case is that of incompressible flow where we take a source of water emanating from the origin of coordinates and spreading out in a circularly symmetric pattern. The initial flow is assumed to exist from very close to $r=0$ out to $R$, which is assumed to be larger than any other length scales in the problem. We then take the limit as $R \rightarrow \infty$, in the same spirit as other studies of this initial-value problem on infinite intervals [2]. The speed must decrease as $r^{-1}$ to conserve mass in an initially steady flow. In sum,

$$
u(r, 0)=u_{0} \frac{r_{0}}{r}, \quad r_{0} \leq r \leq R,
$$

so that (25) integrates to

$$
\theta_{0}=k_{0}\left(\frac{r_{0}}{r}\right)^{a}
$$

where

$$
a=\frac{u_{0} r_{0}}{2 \nu}
$$

Clearly, $u_{0}$ may be large at the small value $r_{0}$ in such a way that the product $u_{0} r_{0}$ is well defined. Equation (29) can now be integrated using Hankel's generalization of (27) as discussed in Watson [21], so that

$$
\theta=k_{0} r_{0}^{a} \Gamma\left(1-\frac{a}{2}\right)(4 \nu t)^{-a / 2} \sum_{n=0}^{\infty} \frac{\left(\frac{a}{2}\right)_{n}}{(n !)^{2}}\left(-\frac{r^{2}}{4 \nu t}\right)^{n}
$$

where $\left(\frac{a}{2}\right)_{n}$ is Pochhammer notation,

$$
\left(\frac{a}{2}\right)_{n}=\frac{a}{2}\left(\frac{a}{2}+1\right)\left(\frac{a}{2}+2\right) \cdots\left(\frac{a}{2}+n-1\right)
$$

and

$$
\left(\frac{a}{2}\right)_{0}=1 .
$$

The full nonlinear solution can now be derived from (19) and expressed in terms of the confluent hypergeometric function as

$$
u(r, t)=\frac{a}{2} \frac{r}{t} \frac{M\left(a / 2+1,2,-r^{2} /(4 \nu t)\right)}{M\left(a / 2,1,-r^{2} /(4 \nu t)\right)}
$$

or more clearly as

$$
u(r, t)=\frac{r}{t} \frac{\sum_{n=1}^{\infty}\left(\frac{a}{2}\right)_{n} \frac{n}{(n !)^{2}}\left(-\frac{r^{2}}{4 \nu t}\right)^{n-1}}{\sum_{n=0}^{\infty} \frac{1}{(n !)^{2}}\left(\frac{a}{2}\right)_{n}\left(-\frac{r^{2}}{4 \nu t}\right)^{n}} .
$$


Isochrones of Nonlinear Solution $u(r, t)$

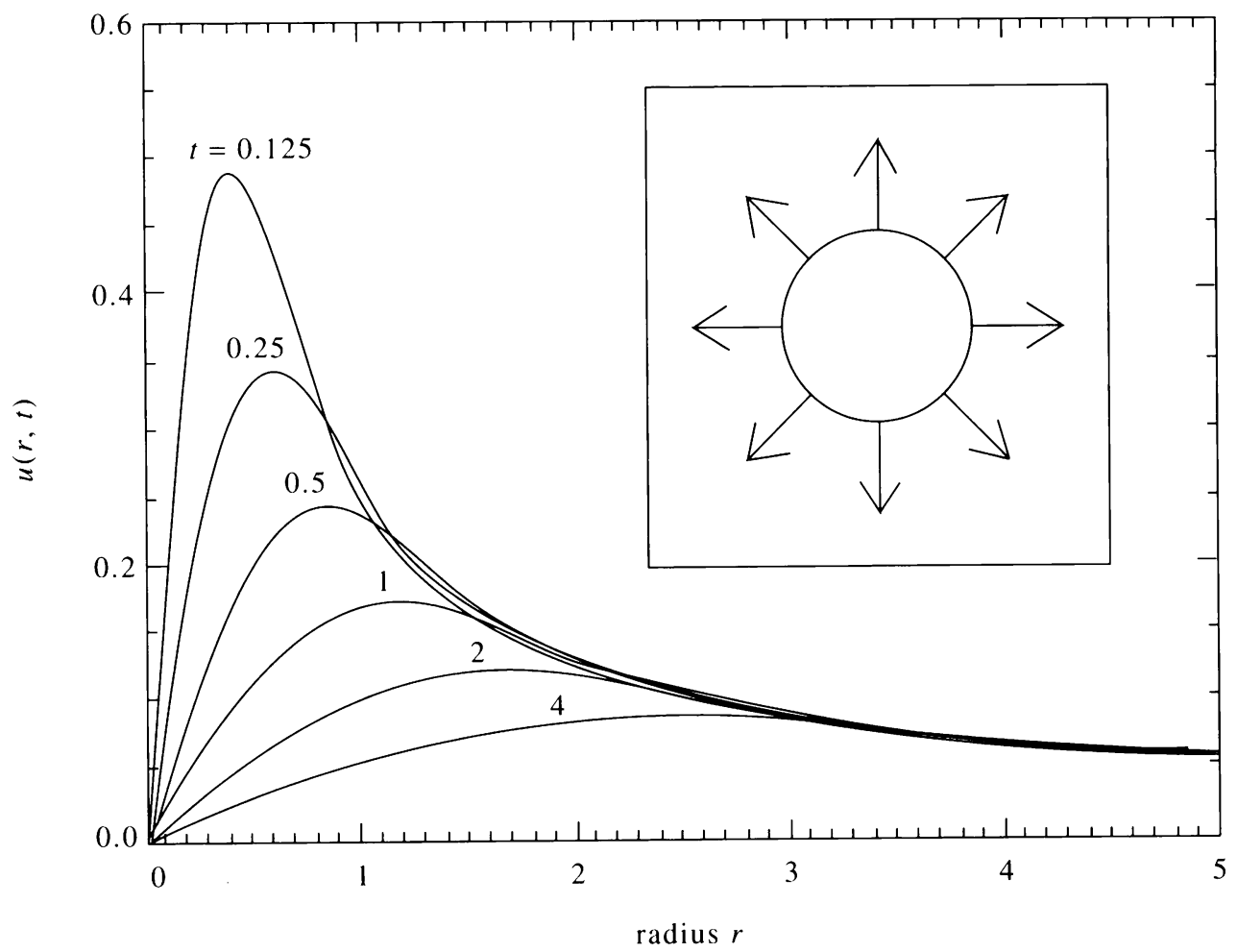

FIG. 1. The nonlinear evolution of a wind with an initial spatial dependence of $1 / r$. The isochrones show the decay of the wind, and, especially, the diffusion at the leading edge where the wind was turned off as this discontinuity propagated outward.

We see that the solution can be written as the product of the inviscid solution times an infinite sum of powers of the similarity variable $r^{2} /(4 \nu t)$. We note the nonlinear effect on higher powers of the similarity variable through the occurrence of the factor $n$ in the numerator. The transient nonlinearity steepens but diffusion dominates; note that the transient is not by itself a solution of Burgers' equation since we do not have the superposition theorem of linear theory.

Figure 1 was created by nondimensionalizing Eq. (17) in the usual fashion, i.e., $r \rightarrow r / L, t \rightarrow \nu t / l^{2}, u \rightarrow u / U$. Choosing $U=u_{0}$ and $L=r_{0}$, then $a$ is 0.5 . This ratio of series is initially divergent and required 100 terms in double precision on a Sun SPARCstation. The solutions are presented as isochrones, showing the evolution of the nonlinear solution towards a steady-state. The boundary where the wind was turned off moves outward while diffusion smooths out the discontinuity. The wind initially drops off as $1 / r$ so that the peak in the profile approaches the starting radius for the source as $t \rightarrow 0$. All curves approach the steady-state envelope of $1 / r$ while diffusion flattens the leading edge. Asymptotically the velocity is zero everywhere.

B. The decay of an initially linearly increasing wind. Example two is a wind emanating from the origin, again, but now the velocity linearly increases in magnitude 
out to some radius, $r_{0}$, chosen to be larger than any other length scale in the problem. We had in mind the initial theoretical treatments of radiatively-driven winds [6] from early-type stars, but our description is too simple to be applied to this problem. This is because of our assumption of cylindrical rather than spherical symmetry, and also because other effects are now known to be important in these winds [8].

The driving force is imagined to turn off at $t=0$, and then we calculate the decay of the flow. In sum

$$
u(r, 0)=u_{0} \frac{r}{r_{0}}=\frac{r}{t_{0}}, \quad 0 \leq r \leq r_{0} ; \quad t=0
$$

where

$$
t_{0}=\frac{r_{0}}{u_{0}}
$$

is half the time for the wind to flow from the source to the initial outer boundary. Both $r_{0}$ and $u_{0}$ are large, but $t_{0}$ is not necessarily big. Then

$$
\begin{gathered}
\theta_{0}=k_{0} e^{-b r^{2}} \\
b=\frac{u_{0}}{4 \nu r_{0}}
\end{gathered}
$$

Proceeding as before,

$$
\begin{gathered}
\theta=\frac{k_{0}}{1+t / t_{0}} \exp \left[-\frac{r^{2}}{4 \nu t} \frac{1}{1+t_{0} / t}\right], \\
u(r, t)=\frac{r}{t+t_{0}} .
\end{gathered}
$$

We note that the solution approaches the Burgers' sawtooth shape as $t \rightarrow \infty$.

5. Conclusions. We have given a relatively simple proof that Burgers' equation can be solved for curvilinear coordinate systems in three dimensions by assuming irrotational flow and using Eq. (16) with $\theta$ derived from solutions of the diffusion equation. We solved two examples in cylindrical coordinates with circular symmetry to show how to implement the generalized solution. Our goal has been to extend the tools of workers who model the complicated interplay between nonlinear steepening and viscous diffusion in waves.

Acknowledgments. ZEM acknowledges partial support of this work by the NSF under grant no. ATM-9119580. EJS acknowledges partial support of this work under grant no. NAG 2001 from NASA-Goddard Space Flight Center.

\section{REFERENCES}

[1] H. Bateman, Some recent researches on the motion of fluids. Monthly Weather Rev. 43, 163 (1915)

[2] E. R. Benton, Solutions illustrating the decay of dissipation lavers in Burgers' nonlinear diffusion equation, Phys. Fluids 10, 2113 (1967)

[3] E. R. Benton, A table of solutions of the one-dimensional Burgers equation, Quart. Appl. Math. 30, 195-212 (1972)

[4] J. M. Burgers, Application of a model system to illustrate some points of the statistical theory of free turbulence. Proc. Roy. Neth. Acad. Sci. Amsterdam 43, 2-12 (1940) 
[5] J. M. Burgers, The Non-Linear Diffusion Equation, D. Reidel Pub. Co., Dordrecht-Holland, 1974

[6] J. I. Castor, D. C. Abbott, and R. I. Klein, Radiatively-driven winds in Of stars, Astrophys. J. 195, 157 (1975)

[7] J. D. Cole, On a quasi-linear parabolic equation occurring in aerodynamics, Quart. Appl. Math. 9, 225-236 (1951)

[8] D. B. Friend and D. C. Abbott, The theory of radiatively-driven stellar winds. III. Wind models with finite disk correction and rotation, Astrophys. J. 311, 701 (1986)

[9] Z. A. Gol'berg, Finite amplitude waves in magnetohydrodynamics, JETP 15, 179 (1962)

[10] S. N. Gurbatov, A. I. Saichev, and S. F. Shandarin, The large-scale structure of the universe in the frame of the model of non-linear diffusion, Monthly Notices Roy. Astronom. Soc. 236, 385 (1989)

[11] S. N. Gurbatov, A. N. Malakhov, and A. I. Saichev, Nonlinear Random Waves and Turbulence in Nondispersive Media: Waves, Rays, and Particles, Manchester University Press, distributed by St. Martin's Press, New York, 1991

[12] E. Hopf, The partial differential equation $u_{t}+u u_{x}=\mu u_{x x}$, Comm. Pure and Appl. Math. 3, 201-230 (1950)

[13] D. T. Jeng, R. Foerster, S. Haaland, and W. C. Meecham, Statistical initial-value problem for Burgers' model equation of turbulence, Phys. Fluids 7, 2114 (1966)

[14] J. I. Katz and M. L. Green, A Burgers model of interstellar dynamics, Astronom. and Astrophys. 161 (1986)

[15] L. A. Kofman and S. F. Shandarin, Theory of adhesion for the large-scale structure of the universe, Nature 334, 129 (1988)

[16] L. A. Kofman and A. C. Raga, Modeling structures of knots in jet flows with the Burgers equation, Astrophys. J. 390, 359 (1992)

[17] P. Lagerstrom, J. D. Cole, and L. Trilling, Problems in the Theory of Viscous Compressible Fluids, monograph, California Institute of Technology, 1949

[18] M. J. Lighthill, Viscosity effects in sound waves of finite amplitude, in Surveys in Mechanics (Batchelor and Davies, eds.), Cambridge Univ. Press, Cambridge, 1956

[19] H. Margenau and G. M. Murphy, The Mathematics of Physics and Chemistry, Von Nostrand Co., London, 1956

[20] B. Van Der Pol, On a non-linear partial differential equation satisfied by the logarithm of the Jacobian theta-functions, with arithmetical applications, Proc. Acad. Sci. Amsterdam A13, 261-271, 272-284 (1951)

[21] G. N. Watson, A Treatise on the Theory of Bessel Functions, second ed., Cambridge Univ. Press, Cambridge, 1962, pp. 393-396

[22] K. B. Wolf, L. Hlavaty, and S. Steinberg, Non-linear differential equations as invariants under group action on coset bundles: Burgers and Korteweg-de Vries equation families, J. Math. Anal. Appl. 114, 340 (1986) 